\title{
Estudio sobre el cambio de elección de tratamiento renal sustitutivo en pacientes que han optado por diálisis peritoneal
}

\author{
Garrido López, María del Valle* \\ Sesmero Ramos, Carolina* \\ Portolés Pérez, José María**
}

* Diplomada Universitaria de Enfermería

**Nefrólogo

Unidad de Diálisis

Servicio de Nefrología

Fundación Hospital Alcorcón (Madrid)

\section{RESUMEN}

La elección de tratamiento renal sustitutivo es una decisión crucial en los pacientes con insuficiencia renal crónica. De ahí la importancia de la información que les ofrecemos en la consulta de prediálisis. Dentro del grupo de pacientes que optan por diálisis peritoneal (DP) como terapia renal sustitutiva (TRS), existen algunos, que por propia voluntad, cambian esta decisión y pasan a hemodiálisis (HD), ya sea en situación de prediálisis, o incluso estando ya en diálisis peritoneal.

Hemos analizado la elección de TRS en nuestra área de salud, características de los pacientes, la prevalencia y variables que influyen en el cambio de técnica (desde DP a HD), determinando el perfil del paciente más susceptible de cambiar su decisión.

Los resultados ponen de manifiesto que la familia tiene una elevada influencia en el cambio de opción. La principal preocupación de todos los pacientes que eligen DP, es el miedo a la peritonitis, tanto si posteriormente cambian su elección como si no lo hacen. Una queja común es la falta de espacio para almacenar el material. Otras características importantes son: el hecho de que consideran la técnica complicada (36.4\%), que precisan colaboración de alguien para realizarla (27.3\%) y que tienen una presión familiar negativa hacia la técnica (27.3\%).

\section{Correspondencia:}

María del Valle Garrido López

Auda de las Retamas $n^{\circ} 79,1^{\circ} \mathrm{A}$

28922 Alcorcón (Madrid)
PALABRAS CLAVE: ELECCIÓN DE TÉCNICA

CAMBIO DE TÉCNICA

DIÁLISIS PERITONEAL

PREDIÁLISIS

STUDY ON THE CHANGE IN CHOICE OF RENAL REPLACEMENT TREATMENT IN PATIENTS WHO HAVE OPTED FOR PERITONEAL DIALYSIS

\section{SUMMARY}

The choice of renal replacement treatment is a crucial decision in patients with chronic renal failure. Hence the importance of the information we offer them in the pre-dialysis consultation. Within the group of patients who opt for peritoneal dialysis (PD) as a renal replacement therapy (RRT), there are some who at their own choice change this decision and move onto haemodialysis (HD), either at the pre-dialysis phase or even after having started peritoneal dialysis.

We have analysed the choice of RRT in our health area, patient characteristics, prevalence and the variables that influence the decision to change technique (from PD to HD), determining the profile of patients most likely to change their mind.

The results show that the family has a significant influence on the change in option. The main concern of all patients who choose PD is the fear of peritonitis, both if they change their mind and if they do not. A common complaint is the lack of space to store the material. Other important 
characteristics are: the fact that it is considered a complicated technique (36.4\%), that it requires the collaboration of another person (27.3\%) and that there is pressure from the family against using this technique (27.3\%).

KEYWORDS: CHOICE OF TECHNIQUE

CHANGE OF TECHNIQUE

PERITONEAL DIALYSIS

PRE-DIALYSIS

\section{INTRODUCCIÓN}

La elección de técnica renal sustitutiva (TRS) en pacientes con insuficiencia renal terminal (IRT) es una decisión crucial, porque influirá posteriormente en el estilo y calidad de vida del paciente así como en el de sus cuidadores. El fracaso o éxito de la diálisis peritoneal va a depender en gran medida de la aceptación de la enfermedad, de la elección de técnica por el propio paciente y del apoyo familiar y profesional recibido.

Esta decisión debe realizarse de forma informada y conjuntamente con sus cuidadores, como se acordó en la Conferencia de Munich de 1990. La Sociedad Europea de Diálisis y Transplante Renal acuerda: "Todos los pacientes con fracaso renal crónico deben ser informados de las distintas opciones terapéuticas, para que ellos mismos puedan escoger". Por otra parte, la Ley 41/2002, 14 de noviembre de autonomía del paciente, recoge el derecho del paciente a la participación informada en la toma de decisiones.

El momento y la calidad de la asistencia prediálisis se reconoce actualmente como un factor determinante en la morbilidad y calidad de vida de los pacientes. Desde la apertura de nuestra unidad, disponemos de consulta de enfermería prediálisis. Entre sus funciones incluye la información sobre las opciones terapéuticas a los pacientes y el asesoramiento ante la elección de TRS.

Una vez que el paciente está en situación de tener que elegir tratamiento renal sustitutivo ya sea la hemodiálisis (HD) o la diálisis peritoneal (DP), podemos clasificar a los pacientes en:

1. Pacientes en los que uno de los dos tipos de TRS está contraindicado, de forma que deben obligatoriamente elegir el otro tipo.

2. Pacientes que expresan de manera clara, sin dudas su decisión, ya sea $\mathrm{DP}$ o $\mathrm{HD}$.

3. Pacientes que en principio se inclinan por $\mathrm{HD}$, pero luego deciden modificar su elección.

4. Pacientes que en principio se inclinan por DP, pero luego modifican su elección.
En este estudio analizaremos la elección de TRS y las razones que llevan a este cuarto grupo de pacientes a modificar dicha elección.

Trabajos previos consideran que la transferencia de DP a HD se debe en un 13\% a problemas sociales y en un $6 \%$ a decisión del paciente. Por otro lado el $60 \%$ de los pacientes que cambian a HD sufren deficiencias físicas, manuales, visuales u otras.

No podemos olvidar que la DP es una técnica de autocuidado y domiciliaria, los pacientes o sus cuidadores, en el caso que tuvieran que hacerse cargo de la técnica, deben valorar muy bien si pueden o deben asumir la responsabilidad de este tratamiento. Además debemos tener en cuenta que la edad es un factor importante a la hora de adquirir conocimientos y esto es imprescindible en DP.

\section{OBJETIVOS}

- Describir la elección de TRS en el área 8 de Madrid y la prevalencia de pacientes que cambian de DP a HD por decisión propia.

- Analizar las características de estos pacientes y los factores implicados en esta modificación en la elección de TRS.

\section{MATERIAL Y MÉTODO}

Realizamos un estudio retrospectivo de los pacientes valorados en la unidad de prediálisis de la Fundación Hospital Alcorcón desde su apertura en enero de 1999 hasta junio de 2004. La muestra del estudio está constituida por 70 pacientes subdivididos en dos grupos:

El primer grupo incluye pacientes que cambian de elección de técnica (grupo CT) e incluye:

- Pacientes de prediálisis que eligieron DP como TRS y que antes de entrar en la técnica modificaron su elección.

- Pacientes ya incluidos en programa de DP que decidieron, por propia voluntad, pasar a HD.

El segundo grupo incluye pacientes que eligieron DP como TRS y no modificaron dicha elección en ningún momento (grupo NCT).

Se excluyeron pacientes que por criterios médicos su única alternativa de TRS fue la HD y los pacientes de prediálisis que eligieron HD y en ningún momento han modificado su elección. 
Se han recogido de la historia clínica electrónica y de la valoración específica de prediálisis de Enfermería los siguientes datos:

1. Variables demográficas (edad, sexo)

2. Distribución de la procedencia de los pacientes en DP (HD, prediálisis o de trasplante)

3. Elección de la técnica: estudiamos si los pacientes iniciaron DP por propia elección o por indicación médica.

Mediante un cuestionario específico se analizaron las causas de modificación de elección de TRS:

1. Presión negativa de los cuidadores hacia DP

2. Necesidad de colaboración familiar para la realización de la técnica de DP

3. Percepción de la técnica como muy complicada

4. No llegar a entender la técnica

5. Miedo a las infecciones

6. Desconfianza hacia la técnica

7. Sensación de mayor seguridad con un tratamiento hospitalario

8. Falta de espacio para almacenar el material

9. Presencia de animales en domicilio

10. Limitaciones físicas en el paciente

11. Percepción del paciente como una carga para sus cuidadores

12. Recibieron información positiva hacia HD

13. Recibieron información negativa hacia la DP

14. Consideran venir al hospital como un entretenimiento

Los resultados fueron procesados en una base de datos ACCESS, y fueron analizados a través del paquete estadístico SPSS, . Se utilizaron media, Chi cuadrado de Pearson c2 y estadístico exacto de Fisher para el análisis de variables cualitativas y cuantitativas según procediese. Se consideró el límite 0.05 para significación estadística.

\section{RESULTADOS}

Se han estudiado un total de 70 pacientes que eligieron DP, de los cuales un $53 \%$ eran varones, con una edad media a la entrada en diálisis de 52 años \pm 12 años (rango 22-85). De estos, 46 pacientes (65.7\%) procedían de prediálisis, 22 de HD (31.42\%) y tan sólo dos (2.85\%) de trasplante fallido.

Todos los pacientes que eligieron DP como primera técnica, lo hicieron por propia elección, en ningún caso se indicó entrada en DP por causa médica. Todos los pacientes que inician DP post trasplante lo hicieron por propia elección y en todos los casos la técnica previa al trasplante había sido la HD.
Los pacientes de DP procedentes de HD que cambiaron de técnica por indicación médica fueron 7, por los siguientes motivos: intolerancia a las sesiones de HD (2), alergia a la heparina (1), e imposibilidad de acceso vascular estable (4).

El grupo (CT) está constituido por 11 pacientes (15.7\% de los pacientes estudiados). De ellos el $55 \%$ eran varones, con una edad media a la entrada en DP de 65 años \pm 5 años, (rango 33-83). El cambio de elección de técnica se produjo en 6 de ellos en prediálisis, en 1 durante el entrenamiento y en 4 tras más de un mes en DP. Existe una presión familiar negativa hacia la decisión de DP en 3 casos. Precisaban colaboración familiar para la realización de la técnica en 3 y en 2 de los casos coexistían ambas características.

El grupo NCT está constituido por 59 pacientes, el $56 \%$ (33) varones y la edad media en el momento de la elección de la técnica fue de 52 años \pm 6 años, (rango 22-85). En este grupo existía una presión familiar negativa hacia la DP en $3 \mathrm{ca}-$ sos; precisaban colaboración familiar para la realización de la técnica en 11 y en 3 coexistían ambas características.

La tabla 1 recoge los elementos asociados a la decisión de cambiar de técnica; cada paciente puede señalar más de un motivo.

\begin{tabular}{|lccc|}
\hline $\begin{array}{l}\text { VARIABLE } \\
\begin{array}{l}\text { Presión negativa de los } \\
\text { cuidadores hacia la DP }\end{array}\end{array}$ & $33 \%$ & $5.08 \%$ & 0.045 \\
\hline $\begin{array}{l}\text { Necesidad de colaboración } \\
\text { familiar técnica DP }\end{array}$ & $33 \%$ & $18.64 \%$ & 0.681 \\
\hline $\begin{array}{l}\text { Percepción de técnica DP } \\
\text { muy complicada }\end{array}$ & $40 \%$ & $6.77 \%$ & 0.018 \\
\hline No llegó a entender técnica DP & $4 \%$ & $0 \%$ & 0.157 \\
\hline Miedo a infecciones & $52 \%$ & $42.37 \%$ & 0.520 \\
\hline Desconfianza hacia la técnica & $20 \%$ & $0 \%$ & 0.023 \\
\hline $\begin{array}{l}\text { Sensación de mayor seguridad } \\
\text { con tratamiento hospitalario }\end{array}$ & $8 \%$ & $1.69 \%$ & 0.292 \\
\hline $\begin{array}{l}\text { Falta de espacio para almacenaje } \\
\text { de material }\end{array}$ & $36 \%$ & $49.15 \%$ & 0.435 \\
\hline Existencia de animales en domicilio & $4 \%$ & $11.9 \%$ & 1.000 \\
\hline $\begin{array}{l}\text { Existencia de limitaciones físicas } \\
\text { del paciente }\end{array}$ & $28 \%$ & $15.25 \%$ & 0.386 \\
\hline $\begin{array}{l}\text { Percepción del paciente como } \\
\text { una carga para sus cuidadores }\end{array}$ & $20 \%$ & $18.64 \%$ & 1.000 \\
\hline $\begin{array}{l}\text { Recibieron información positiva } \\
\text { hacia la HD }\end{array}$ & $4 \%$ & 0.157 & 0.157 \\
\hline $\begin{array}{l}\text { Recibieron información negativa } \\
\text { hacia la DP }\end{array}$ & $4 \%$ & $0 \%$ & 0.157 \\
\hline $\begin{array}{l}\text { Consideran venir a hospital como } \\
\text { entretenimiento }\end{array}$ & $4 \%$ & $0 \%$ & 0.157 \\
\hline
\end{tabular}

Tabla 1: Comparación de los elementos que influyen en la toma de decisión de cambio de técnica entre el grupo CT y el grupo NCT. 
Se estudió la asociación entre distintos motivos, destacando una relación significativa $(p=0.0001)$ entre colaboración familiar para realización de la técnica y limitaciones físicas del paciente. Así mismo se observó que no existía relación entre la sensación de carga familiar y la falta de espacio doméstico $(p=0.760)$.

Si evaluamos la presencia combinada de items, se observó en 10 casos (28.6\%) que el paciente se considera una carga familiar y precisa de colaboración para realizar la técnica. Existe relación entre la necesidad de colaboración familiar para la realización de la técnica y la existencia de limitaciones físicas del paciente en 8 casos $(66,7 \%)$, en los que el paciente precisa de colaboración familiar para realizar la técnica debido a una limitación física. En 4 casos (28.6\%) el paciente precisa de colaboración familiar, pero existe una presión negativa por parte de la familia a asumir los cuidados que conlleva la DP. En 7 casos $(21,2 \%)$ el paciente cree que es una carga para la familia, además de existir una falta de espacio para almacenar material.

\section{DISCUSIÓN}

Los pacientes que eligieron DP o incluso inician la técnica y después deciden cambiar a HD son 11 (15.7\%), de los cuales 6 son pacientes en prediálisis, que ni siquiera llegan a comenzar la técnica. Debemos plantearnos si es posible reducir esta cifra, mejorando la intervención de enfermería tanto en la consulta prediálisis en el momento de elegir TRS como posteriormente.

Con todos los datos obtenidos hemos definido un perfil del paciente que cambia su elección tras elegir DP; su media de edad es de 65 años, frente a los 52 años de media de los pacientes que entran en DP, es decir, tienen más probabilidad de modificar su elección de técnica los pacientes de mayor edad, esto viene asociado a mayor limitación y dependencia del paciente. No existen diferencias significativas en cuanto al sexo. En la mayoría de los casos son pacientes que rechazan la enfermedad, no toman conciencia de ella y no llegan a asumirla.

Una de las características más relevantes es que suelen ser pacientes dependientes que viven en la mayoría de las ocasiones con sus hijos. A la familia el hecho de tener que asumir el papel de cuidador le supone un cambio importante en su forma de vida, surgiendo así una situación de rechazo hacia la técnica. La influencia de la familia es realmente importante, ya que hemos encontrado relación significativa entre presión negativa familiar hacia la DP e índice de pacientes que cambian su elección. En una consulta prediá- lisis se debería estudiar si es suficiente la información y apoyo que recibe no sólo el paciente, sino también los cuidadores e intentar que se sintieran más seguros en el caso de elegir DP.

Hemos observado una tendencia a menos colaboración familiar en el grupo que cambia de idea, aunque no alcanza significación estadística. Al aumentar la edad media de los pacientes, aumenta su dependencia y precisan en muchos casos un cuidador que les preste apoyo o realice la técnica. Esto conlleva una implicación que, en muchas ocasiones no se puede ofrecer, aumentando el riesgo de que el paciente decida abandonar la técnica, o no llegue a comenzarla. Sí es muy significativa la relación entre la necesidad de colaboración familiar para realizar técnica DP y el sentimiento del paciente de ser una carga para la familia. Deberíamos entender que existe un número importante de cuidadores que se está haciendo cargo de un tratamiento domiciliario. La pregunta que deberíamos plantearnos es si estos cuidadores reciben suficiente cuidado $y$ apoyo.

Los pacientes que prueban DP en la mayoría de los casos no la ven como una técnica muy complicada, y además dejan de desconfiar de ella, existiendo diferencias significativas respecto al grupo NCT (que no olvidemos en la mayoría de los casos nunca comenzaron DP).

Llama la atención el miedo de los pacientes a las infecciones, tanto si cambian de técnica como si no. Esto no se corresponde con la incidencia real de esta complicación, que en nuestra área se encuentra en torno a una peritonitis cada 19 meses. Debemos plantearnos si la información que ofrecemos en la consulta prediálisis sobre este punto es objetiva.

Otro factor importante señalado por los pacientes, es la falta de espacio para almacenaje, no olvidemos que en muchos casos, los pacientes conviven con varios miembros de la familia reduciendo el espacio disponible para almacenaje en las viviendas. Debemos reducir al mínimo este problema, con envíos más frecuentes y con menor stock, facilitando de esta forma la elección de DP.

No llega a ser significativa la relación entre limitaciones físicas $e$ índice de cambio de técnica, aunque haya mayor prevalencia de pacientes con limitaciones físicas en el grupo CT. Tampoco llega a ser significativa la relación entre que el paciente crea que es una carga para la familiar la decisión de cambiar de técnica. Creemos que debe existir relación entre estas variables, aunque la prevalencia es similar en ambos grupos. Quizás, esta variable debe estar interferida, pues el grado de dependencia esta influido por otras patologías concomitantes en cada paciente. 
Todos los pacientes que desconfían de la técnica deciden cambiar; por tanto, deberíamos discernir si esta desconfianza es real o enmascara una falta de información y mala comprensión de la DP. Dado que los pacientes que llegan a probar la DP cambian su visión respecto a la complejidad de la misma; deberíamos facilitar el contacto de pacientes prediálisis con pacientes que ya están en técnica DP.

\section{CONCLUSIONES}

- Sería importante mejorar el apoyo a los cuidadores de los pacientes en DP, tanto en la fase prediálisis como en la fase de DP ya que, en la actualidad, en nuestra área, los cuidadores no reciben ningún tipo de soporte.

- La información sobre TRS debe darse de forma clara y objetiva, intentando que el paciente vea cada técnica de manera global, sin miedos.

- Es necesario el refuerzo en prediálisis cuando un paciente se decide por DP; sería importante reforzar su decisión cada vez que venga a consulta, para vincularlos más a la técnica y facilitar contactos con pacientes ya en DP.

- También la industria podría mejorar su relación con los pacientes, teniendo en cuenta los problemas de almacenaje que tienen. Además, habría que seguir desarrollando sistemas para intentar adaptar aún más la técnica al paciente con limita- ciones físicas y de esta manera darles más independencia, liberando a la familia de parte de la carga que asumen.

\section{BIBLIOGRAFÍA}

1. Blasco Cabañas C, Ponz Clemente E, Mañe Buixo N, Martínez Ocaña JC, Marquina Parra D, Yuste Giménez E et al. Estudio detallado de las causas de transferencia de Diálisis Peritoneal a Hemodiálisis en un Servicio de Nefrología. Sociedad Española de Enfermería Nefrológica 2004; 7(1): 43-48.

2. Jofré R. Factores que afectan a la calidad de vida en pacientes en prediálisis, diálisis y trasplante renal. Nefrología 1999; Vol XIX (supl 1): 84-90.

3. Luque Vadillo E, Barroso Ortin R, López Viñals M, Alapont Areste M, Alconchel Cabezas S, Torres Jansá M et al. Diálisis Peritoneal, la realidad subjetiva de convivir con una enfermedad crónica. Sociedad Española de Enfermería Nefrológica 2001; (1): 12-17.

4. Muriana Zamudio C, Puigvert Vilalta C, García Garrido ML. Valoración de un programa de educación sanitaria a pacientes con insuficiencia renal crónica. Sociedad Española de Enfermería Nefrológica 2002; (5): 14-17.

5. Serrato Ruiz F, Ramos García JA, Pérez Garrigues A, Durá Caravaca M, Rovira Clos P. Nuestra experiencia con un protocolo de información sobre opciones de tratamiento en la insuficiencia renal crónica. Comunicaciones del XXVIII Congreso Nacional de la SEDEN. Palma de Mallorca 2003: 205-212. 